Revue d'histoire de l'Amérique française

REVUE D.HISTOIRE DE L'AMÉRIQUE FRANÇAISE

\title{
HOGUE, Clarence, André BOLDUC et Daniel LAROUCHE, Québec, un siècle d'électricité. Montréal, Éditions Libre Expression, 1979. 415 p. \$65.00.
}

\section{Pierre Lanthier}

Volume 34, numéro 3, décembre 1980

URI : https://id.erudit.org/iderudit/303889ar

DOI : https://doi.org/10.7202/303889ar

Aller au sommaire du numéro

Éditeur(s)

Institut d'histoire de l'Amérique française

ISSN

0035-2357 (imprimé)

1492-1383 (numérique)

Découvrir la revue

Citer ce compte rendu

Lanthier, P. (1980). Compte rendu de [HOGUE, Clarence, André BOLDUC et Daniel LAROUCHE, Québec, un siècle d'électricité. Montréal, Éditions Libre Expression, 1979. 415 p. \$65.00.] Revue d'histoire de l'Amérique française, 34(3), 454-457. https://doi.org/10.7202/303889ar d'utilisation que vous pouvez consulter en ligne.

https://apropos.erudit.org/fr/usagers/politique-dutilisation/ 
HOGUE, Clarence, André BOLDUC et Daniel LAROUCHE, Québec, un siècle d'électricité. Montréal, Éditions Libre Expression, 1979. 415 p. $\$ 65.00$

Depuis la parution de la monographie de J.H. Dales sur l'hydroélectricité au Québec de 1898 à 1940, aucune synthèse portant sur ce sujet n'avait été publiée. Le présent ouvrage vient combler ce retard, en reprenant l'étude à partir des origines et en la prolongeant jusqu'à nos jours.

Ce livre est divisé en deux sections. La première, rédigée par Clarence Hogue, remonte aux premières expériences d'éclairage électrique à Montréal en 1878 pour aboutir aux nationalisations de 1962-63: elle balaie cette période avec un faisceau qui s'arrête sur chacune des grandes régions (et compagnies) du Québec. La seconde section, écrite par André Bolduc et Daniel Larouche, ne s'occupe que de l'exploitation publique de l'énergie électrique, depuis la création de l'Hydro-Québec en 1944 jusqu'à l'aménagement de la Baie James.

Ayant eu accès à une importante documentation fournie par l'HydroQuébec et par différentes institutions publiques québécoises et canadiennes, les auteurs ont donné le primat à la description et à une accumulation fort impressionnante de faits, de noms et de dates. De plus, le texte s'accompagne d'une remarquable collection de documents iconographiques, dont certains sont très révélateurs des moments cruciaux de l'histoire hydro-électrique québécoise (l'on songe entre autres à la photographie de la page 286, exposant la mine patibulaire des employés de la Shawinigan Water and Power, contraints, en 1964, d'apprendre le français). Toutefois, la tâche du lecteur aurait été facilitée si l'on avait complété le texte par des tableaux statistiques et des graphiques sur l'évolution physique et financière de l'industrie électrique.

L'histoire événementielle, donc, domine totalement l'ouvrage. Il faut cependant noter que les auteurs n'ont pas décrit les mêmes réalités d'une section à l'autre.

Clarence Hogue met surtout de l'avant trois aspects de l'industrie hydro-électrique: la propriété du capital, les profits (scandaleux de préférence), et la nationalité, sinon le groupe linguistique, des dirigeants. L'auteur voit, dans la plupart des fusions d'entreprises, un accroissement du pouvoir de certains individus, et, dans la majorité des opérations financières, une façon d'augmenter le profit. À elle seule, d'ailleurs, la Montreal Light, Heat and Power semble rassembler tout ce que le capitalisme a de louche et de mesquin. Enfin, Hogue note que presque partout au Québec les premières tentatives de production d'électricité ont été l'oeuvre de Canadiens français. Les Américains et les Canadiens anglais se contentaient de racheter les réseaux, afin de les accroître et de les 
regrouper. Et l'auteur ne manque pas l'occasion de porter un jugement défavorable sur les compagnies où l'élément anglophone domine nettement la partie francophone.

Que les capitalistes cherchent par tous les moyens à faire du profit ne saurait surprendre personne. Plus intéressant aurait été de savoir ce que l'on faisait avec ce profit: le réinvestissait-on dans l'électricité ou dans d'autres secteurs? Au Québec ou ailleurs?

D'autre part, la formation des monopoles ne s'explique-t-elle que par le désir d'éliminer la concurrence? Toutes les grandes villes du monde ont connu, surtout entre 1900 et 1914, de véritables luttes pour l'obtention du monopole de la distribution d'électricité. Or, dans certaines villes (comme Londres avant 1914 et Paris avant 1907), l'absence de monopole signifiait également le manque d'uniformisation du matériel et de la tension du courant, avec tous les désavantages que cela impliquait. Monopoliser la distribution devenait donc une solution logique et, sur ce point, les agissements de la Montreal Light, Heat and Power étaient monnaie courante à l'époque. Cette compagnie persistera-t-elle dans ses pratiques frauduleuses (notamment en matière de comptabilité)? On pourra sans doute n'y voir que de la malhonnêteté. Mais, en deçà de cette attitude immorale, ne pourrait-on pas soupçonner une situation économique difficile ou encore, comme le suggérait Dales, une gestion particulièrement mauvaise? Et en province, les mouvements de concentration n'ont-ils pas eu pour but premier de réaliser l'interconnexion des réseaux et, corollairement, la réduction du nombre des petites centrales, avantageusement remplacées par des unités à forte capacité de production? L'auteur aurait eu avantage à étudier les aspects économiques de l'électricité, et non pas seulement la partie financière ou comptable.

Enfin, faire des dirigeants anglophones, à une ou deux exceptions près, des dominateurs et des profiteurs devient lassant à la longue. Qu'ils aient succédé aux pionniers francophones s'explique non pas par une injustice raciale, mais par le fait que: 1- un réseau électrique n'est rentable qu'à grande échelle; 2 - cela suppose des capitaux en grande quantité, ce que le Canada français ne pouvait offrir à l'époque, vu la faiblesse de ses institutions financières: cette faiblesse interdisait aux investisseurs de prendre des risques dans un domaine aussi nouveau et aussi exigeant en capitaux que l'hydro-électricité. D'ailleurs, tant qu'à faire, l'auteur aurait dû s'étonner d'une aussi forte présence des Canadiens français parmi les pionniers: on nous a tellement peint le Canada français du XIXe siècle comme incapable d'initiative et d'entrepreneurship que cette présence finit par surprendre. D'où venaient tous ces petits entrepreneurs? Et surtout, d'où émanait leur dynamisme?

En fait, l'étude aurait beaucoup gagné à être élargie. Hogue aurait. pu toucher à la question du travail, de même qu'à l'organisation des 
entreprises. Ainsi, la Royal Electric Co. a cédé, en 1900, ses ateliers de construction de matériel électrique à la Canadian General Electric. Hogue voit dans cette opération un accroissement de l'emprise américaine sur le Canada (p. 61). Or, d'une part, la Royal Electric exploitait les brevets des groupes américains Thomson-Houston et Edison; et d'autre part, la General Electric américaine est née de la fusion en 1892 de ces deux groupes. Donc, plutôt que d'accroissement, ne s'agit-il pas ici de spécialisation des activités américaines dans le secteur manufacturier canadien? Il serait intéressant de savoir pourquoi. Par ailleurs, l'une des caractéristiques majeures de l'industrie hydro-électrique privée au Québec, si l'on excepte les investissements de la Shawinigan Water and Power du côté de la chimie, semble avoir été la tendance à la concentration horizontale. Pareille évolution n'entre-elle pas en contradiction avec le processus d'intégration verticale et de formation d'unités multidivisionnelles que l'on observe dans l'ensemble des grandes entreprises nordaméricaines? À quoi rattacher ce mouvement? À la réalité économique québécoise ou à l'industrie hydro-électrique en particulier?

La section couverte par Bolduc et Larouche aborde les activités de

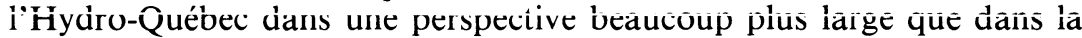
précédente. Alors que Hogue examine les sociétés privées de l'extérieur (du point de vue du simple usager ou, tout au plus, du petit actionnaire), ces deux auteurs nous font connaître la vie interne de l'Hydro-Québec et de la SDBJ. En effet, il y est non seulement question des grands projets comme Bersimis, Manic-Outardes ou la Baie James, mais encore des débats soulevés par les nationalisations et les modalités d'indemnisation, des difficiles problèmes suscités par l'uniformisation du matériel électrique et surtout du personnel des anciennes compagnies, de même que de toutes les questions syndicales depuis la lutte entre la CSN et l'UNESP en 1962-1963 jusqu'aux incidents survenus à la Baie James en 1974, et des nombreux mouvements régionaux de contestation s'attaquant à la validité de certains projets de l'Hydro-Québec. L'ensemble est fort bien documenté et le récit limpide. On regrettera seulement que les auteurs n'aient pas cherché à présenter de façon systématique les résultats financiers de l'Hydro-Québec. Une étude du rendement aurait permis de se prononcer sur le rôle que certains aiment donner aux entreprises publiques dans le capitalisme moderne: oui ou non, ces sociétés n'existentelles que pour sauver le capitalisme de la baisse tendancielle du taux de profit?

Par ailleurs, les luttes syndicales et les contestations locales montrent clairement que l'Hydro-Québec ne joue pas un rôle exclusivement économique dans la province: tout en réassurant les Québécois sur leur savoir-faire économique ("après tout on est capable» - p. 299), dans quelle mesure la Société n'a-t-elle pas contribué, à l'instar d'autres institutions publiques (dont la Société Radio-Canada), à la formation d'une 
nouvelle classe moyenne au Québec, moins boutiquière et paroissiale, beaucoup plus professionnelle et "étatiste»? C'est du moins ce que laissent entendre les grands mouvements de syndicalisation des ingénieurs dans les institutions publiques en 1963 (p. 328).

D'ailleurs, le présent ouvrage constitue en lui-même un fidèle reflet de cette mutation sociale et idéologique: en passant d'une section à l'autre et, donc, du premier des auteurs aux suivants, ne voit-on pas se succéder deux mentalités fort distinctes, celle de la petite bourgeoisie canadienne-française méfiante vis-à-vis de l' "étranger», et celle de la bureaucratie québécoise, fière de ce qu'elle a accompli, mais accablée par les lourdeurs administratives qui créent une distance entre elle et la population? 